COMUNICAÇÃO CIENTÍFICA

\title{
CARACTERIZAÇÃO FÍSICA E FÍSICO-QUÍMICA DE FRUTOS DE DUAS VARIEDADES DE TAMARILHO ORIUNDAS DO NORTE DE MINAS GERAIS ${ }^{1}$
}

\author{
LÍLIAN PANTOJA ${ }^{2}$, NÍSIA ANDRADE VILLELA DESSIMONI PINTO ${ }^{3}$, CRISTIANE LOPES ${ }^{4}$, \\ RENATA GANDRA ${ }^{4}$, ALEXANDRE SOARES DOS SANTOS $^{5}$
}

RESUMO-O tamarilho (Solanum betaceum) é uma espécie de clima subtropical explorado comercialmente como fruto de exportação em países como a Colômbia e Nova Zelândia. No Brasil, esse fruto tem caráter exótico, e suas características nutricionais e tecnológicas são pouco conhecidas. O presente trabalho teve por objetivo caracterizar frutos de duas variedades de tamarilho, amarela e vermelha, oriundos da região do Vale do Jequitinhonha no Estado de Minas Gerais. Foram analisados os aspectos físicos, físico-químicos e a composição centesimal. Os resultados da caracterização centesimal e físico-química mostraram haver uma grande semelhança entre as duas variedades, excetuando o percentual de cinzas $(0,152 \pm 0,046$ e $1,054 \pm 0,339)$ e de açúcares redutores $(5,283 \pm 0,463$ e $2,979 \pm 0,090)$. O rendimento das polpas foi superior a $67 \%$, o valor médio de sólidos solúveis totais foi da ordem de $12,5 \%$ e os valores da relação SST/AcT foi superior a 7,0. Tais medidas permitem considerar o tamarilho um fruto com potencial para o consumo in natura e possível matéria-prima para a indústria de alimentos.

Termo para indexação: tamarilho, tomate de árvore, Solanum betaceum, Cyphomandra betacea, composição centesimal.

\section{PHYSICAL AND PHYSICOCHEMICAL CHARACTERIZATION OF FRUITS OF TWO VARIETES OF TAMARILLO ORIGINATED FROM THE NORTH OF MINAS GERAIS}

\begin{abstract}
The tamarillo (Solanum betaceum) is a subtropical species exploited as a fruit for exportation in countries such as Colombia and New Zealand. In Brazil, this fruit is exotic and their nutritional characteristics and technology are little known. This study aimed to characterize the fruits of two varieties of tamarillo, the yellow and the red, found in the Vale do Jequitinhonha region in the state of Minas Gerais. It was discussed the physical, physicochemical and proximate composition. The results of proximate composition and physicochemical characterization showed that there is a great similarity between the two varieties, presenting only major difference in the percentage of ash $(0.152 \pm 0.046$ and $1.054 \pm 0339)$ and reducing sugars $(5.283 \pm 0.463$ and $2.979 \pm 0.090)$. The yield of tamarillo pulp was more than $67 \%$, the average value of total soluble solids was in order of $12.5 \%$, and the average of the quotient from TSS / TAc was above 7.0. These characteristics of the tamarillo allow considering it for consumption in natura and possible raw material for the food industry.
\end{abstract}

Index Terms: tamarillo, tree tomato, Solanum betaceum, Cyphomandra betacea, proximate composition.

\footnotetext{
'(Trabalho 125-08). Recebido em: 26-05-2008. Aceito para publicação em: 04-05-2009.

${ }^{2}$ Bióloga, Pesquisadora DCR-FAPEMIG, Departamento de Nutrição, FCBS/UFVJM. Rua da Glória, 187, Diamantina-MG. CEP $39100-000$. e-mail:1.pantoja@ufvjm.edu.br

${ }^{3}$ Bióloga, Prof. Adjunto, Depto de Nutrição, FCBS/UFVJM. Rua da Glória, 187, Diamantina-MG. CEP 39100-000 e-mail: nisiavillela@yahoo.com.br

${ }^{4}$ Nutricionista, FCBS/UFVJM. Rua da Glória, 187, Diamantina-MG. CEP 39100-000. lopes.co@hotmail.com ; renatagandra@hotmail.com

${ }^{5}$ Farmacêutico, Prof. Adjunto, Depto de Ciências Básicas, FCBS/UFVJM. Rua da Glória, 187, Diamantina-MG. CEP 39100-000 e-mail: alexandre.soares@ufvjm.edu.br
} 
O tamarilho, Solanum betaceum (Cav.), antes denominado Cyphomandra betacea (Cav.) Sendtn. (Bohs, 1995; Bohs and Olmstead, 1997), pertence à família Solanaceae. No Brasil é conhecido popularmente como tomate de árvore ou tomate francês. Existem descritas três variedades de tamarilho: amarela, vermelha e roxa. A variedade amarela apresenta casca amarelo-brilhante com estrias longitudinais de coloração verde-escura a marrom e polpa amarela. A variedade vermelha possui casca laranja-avermelhada com estrias longitudinais de coloração verde-escura a marrom e polpa amarela a laranja. A variedade roxa apresenta casca vermelho-escura intensa com estrias verticais verdes e polpa laranja-clara (Boyes \& Strübi, 1997; Prohens \& Nuez, 2005). Encontrado em regiões de clima subtropical, o tamarilho é originário da América do Sul, com evidências para áreas do sul da Bolívia e norte da Argentina (Prohens $\&$ Nuez, 2005). Atinge a maturidade após 3 anos de plantio e tem uma vida produtiva de cerca de 7 a 8 anos (Clark \& Richardson, 2002). Em países como Colômbia e Nova Zelândia, tem importância comercial e é produto de exportação. No Brasil, é cultivado basicamente em quintais, principalmente na Bahia, Minas Gerais e São Paulo (Agroforestrytree, 2007). Em Diamantina-MG, essa espécie é encontrada em abundância em quintais, mesmo sem tratos culturais adequados.

$\mathrm{O}$ fruto pode ser consumido in natura, em saladas, como sobremesa, como aperitivo, na forma de suco e ainda, em combinação com outros produtos, como sorvete, leite e iogurte. Por conter alto teor de pectina e por apresentar características desejáveis para a produção de polpa, molhos, comida de bebê e geleias, pode ser explorado comercialmente pela indústria de alimentos (Prohens \& Nuez, 2005). Além disso, é rico em licopeno, vitaminas A, B, C, minerais, como fósforo, potássio e cálcio, e frutose (Boyes \& Strübi, 1997).

No Brasil, o potencial nutricional, as formas de aproveitamento e estudos científicos sobre as cultivares de tamarilho são escassos ou pouco difundidos. Indo ao encontro desta lacuna, buscou-se, no presente trabalho, avaliar a composição centesimal e realizar a caracterização física e físico-química de frutos de duas variedades de tamarilho oriundos da região dos Vales do Jequitinhonha, no norte de Minas Gerais.

Os frutos foram colhidos em estádio de amadurecimento comercial, em plantas cultivadas em Diamantina e municípios circunvizinhos, em um raio de $20 \mathrm{~km}$ da coordenada $18^{\circ} 14^{\prime} 37^{\prime \prime}$ sul e 4336'4" leste, à altitude média de 1.268 metros, com índice médio pluviométrico anual de 1.404,7 $\mathrm{mm}$. Após a colheita, foram acondicionados em caixas de plástico e transportados para o laboratório, onde foram selecionados quanto à sanidade, grau de maturação e injúrias. Em seguida, foram lavados em água corrente, sanitizados com hipoclorito de sódio a $200 \mathrm{ppm}$, lavados novamente e secos à temperatura ambiente. Para caracterização física, 20 frutos de cada variedade, escolhidos aleatoriamente, foram pesados em balança semianalítica, e seu comprimento e diâmetro medidos com auxílio de paquímetro digital. A polpa foi obtida após a remoção das peles e sementes dos frutos. O rendimento em polpa foi calculado mediante a média das relações percentuais entre os pesos das polpas obtidos para cada fruto processado e os respectivos pesos dos frutos inteiros. A polpa foi acondicionada em sacos plásticos, com capacidade para $500 \mathrm{~g}$, e armazenada em freezer $\left(-18^{\circ} \mathrm{C}\right)$ para posterior análise. Os procedimentos analíticos para determinação de umidade, proteína total, lipídios, fibra total, carboidratos e cinza foram efetuados segundo a AOAC (1990); pH, acidez total expressa em ácido cítrico $\left(\mathrm{AcT}_{\text {ácido cítrico }}\right)$; teor de sólidos solúveis totais (SST) e relação SST/AcT foram feitos de acordo com as Normas Analíticas do Instituto Adolfo Lutz- IAL (1985); as determinações de açúcares redutores totais (ART) e redutores (AR) foram realizadas de acordo com o método de Somogyi-Nelson, descrito por Southgate (1991). O valor calórico foi calculado, utilizando-se dos fatores de conversão de Atwater: 9 Kcal por g de lipídios, 4 Kcal por g de proteínas e 4 Kcal por $\mathrm{g}$ de carboidratos (De Angelis, 1977).

Observou-se que não houve diferença entre as variedades amarela e vermelha com relação aos valores médios obtidos para o diâmetro $(4,15 \mathrm{~cm})$ e comprimento $(6,10 \mathrm{~cm})$. Estes valores estão próximos dos relatados por Mwithiga et al. (2007), 4 a $5 \mathrm{~cm}$ para diâmetro e 5 a $10 \mathrm{~cm}$ para comprimento. Não foram observadas diferenças significativas nas medidas de massa e rendimento de polpa entre as duas variedades analisadas (Tabela 1 ). O rendimento médio da polpa foi superior a 60 \%, parâmetro importante na determinação da qualidade industrial por influenciar diretamente no rendimento de produtos a serem obtidos, por exemplo, no preparo de sucos. Os valores obtidos na composição centesimal foram semelhantes entre as variedades avaliadas (Tabela 2), sendo que a maior diferença encontrada foi entre os valores de cinzas ou resíduo mineral fixo, cerca de 6,0 vezes superior na variedade vermelha. Os valores referentes ao percentual de umidade, proteína, carboidratos e energia foram próximos dos reportados por Dignan et al. (2004). Já os percentuais de lipídios e fibras totais foram, em média, respectivamente, 3,4 vezes maiores e 1,5 vez menor, comparados aos 
valores encontrados pelos mesmos autores. A polpa de ambas as variedades apresentou alto percentual de umidade, em média $86,5 \%$, uma característica importante na agroindústria, principalmente para o processamento de bebidas. Quando comparado ao tomate cru (Lycopersicon sp.), o tamarilho apresentou valores superiores de carboidratos (3,9 vezes), lipídios (13,1 vezes) e energia (2,6 vezes), e valor $35 \%$ menor de fibra total. Os valores obtidos para acidez total, açúcares redutores totais, pH e SST (Tabela 3) foram semelhantes nas duas variedades de tamarilho, com exceção do valor de açúcares redutores que, na variedade amarela, foi $77 \%$ superior.

Os valores de acidez total e SST encontrados nas variedades estudadas (Tabela 3) estão próximos dos obtidos por Boyes \& Strübi (1997), respectivamente, $1,60 \%$ e $10,5{ }^{\circ}$ Brix para a variedade vermelha e $1,57 \%$ e $10,85^{\circ}$ Brix para a variedade amarela, e 4,0 e 1,9 vezes maiores que os descritos para o tomate cru (Cardoso et al., 2006). O teor de SST é um fator importante na qualidade de frutos, tanto para o seu consumo in natura, como para o processamento industrial, implicando redução no gasto de açúcar, menor tempo de processamento e economia energética. De acordo com Franco \& Landgraf (2001), o pH obtido está dentro da categoria de alimentos muito ácidos $(\mathrm{pH}<4,0)$, fator intrínseco favorável, por inibir o crescimento de bactérias e outros microrganismos. A relação SST/AcT, que representa o equilíbrio entre o gosto doce e ácido, propiciando uma boa avaliação da "doçura" do fruto, foi em média 7,89 $\pm 0,10$ na variedade branca e 7,46 $\pm 0,27$ na vermelha.

Em síntese, as variedades de tamarilho encontradas na região do Alto Vale do Jequitinhonha, no norte de Minas Gerais, apresentaram características físicas e físico-químicas que as qualificam tanto para o consumo in natura, quanto para o processamento industrial. Entre tais características, destacam-se o baixo valor calorífico do fruto, o bom rendimento de polpa e o elevado valor de SST.

TABELA 1 - Valores das medidas de caracterização física das duas variedades de tamarilho.

\begin{tabular}{lcc}
\hline Variáveis & var. Amarela & var. Vermelha \\
\hline Comprimento (cm) & $6,10 \pm 0,33$ & $6,10 \pm 0,51$ \\
Diâmetro (cm) & $4,30 \pm 0,49$ & $4,00 \pm 0,26$ \\
Peso Bruto (g) & $59,70 \pm 11,50$ & $55,20 \pm 11,59$ \\
Peso Polpa (g) & $41,20 \pm 10,60$ & $40,40 \pm 10,49$ \\
Rendimento (\%) & $67,70 \pm 5,14$ & $72,70 \pm 5,84$ \\
\hline
\end{tabular}

TABELA 2 - Valores da composição centesimal das duas variedades de tamarilho encontradas na região do Alto Vale do Jequitinhonha.

\begin{tabular}{lcccc}
\hline \multicolumn{1}{c}{ Variáveis } & var. Amarela & var. Vermelha & Tamarillo* & Tomate Cru* \\
\hline Umidade (\%) & $86,42 \pm 1,26$ & $86,78 \pm 1,97$ & 86,00 & 95,00 \\
Proteína (\%) & $1,68 \pm 0,02$ & $1,78 \pm 0,11$ & 1,50 & 1,00 \\
Lipídio (\%) & $1,22 \pm 0,29$ & $1,42 \pm 0,26$ & 0,30 & 0,10 \\
Cinza (\%) & $0,15 \pm 0,05$ & $1,05 \pm 0,34$ & nd & nd \\
Fibra (\%) & $0,91 \pm 0,03$ & $0,87 \pm 0,02$ & 2,20 & 1,20 \\
Carboidratos (\%) & $9,41 \pm 1,76$ & $9,10 \pm 1,33$ & 9,10 & 1,90 \\
Energia (Kcal\%) & 55,38 & 52,58 & 49,00 & 15,00 \\
\hline
\end{tabular}

* Dignan et al. (2004).

TABELA 3- Média dos valores das análises físico-químicas das duas variedades de tamarilho avaliadas.

\begin{tabular}{lccc}
\hline Variáveis & var. Amarela & var. Vermelha & Tomate Cru* \\
\hline AcT $_{\text {ácido cítrico }}(\%)$ & $1,65 \pm 0,02$ & $1,65 \pm 0,03$ & 0,33 \\
Açúcares Totais (\%) & $7,62 \pm 0,35$ & $6,39 \pm 0,94$ & nd \\
Açúcares Redutores (\%) & $5,28 \pm 0,46$ & $2,98 \pm 0,09$ & nd \\
pH & $3,00 \pm 0,03$ & $3,84 \pm 0,02$ & 4,39 \\
SST ( ${ }^{\circ}$ Brix) & $13,00 \pm 0,20$ & $12,30 \pm 0,46$ & 4,37 \\
\hline
\end{tabular}

Fonte*: Cardoso et al. (2006). 


\section{REFERÊNCIAS}

AGROFORESTRYTREE. A tree species reference and selection guide. Disponível em: $<\mathrm{http}: / / \mathrm{www}$. worldagroforestrycentre.org/Sites/TreeDBS/aft/ speciesPrinterFriendly.asp? $\mathrm{Id}=639>$. Acesso em: 9 jul. 2007.

BOHS, L. Transfer of cyphomandra (solanaceae) and its species to Solanum. Taxon, Utrecht, v.44, n.4, p. 583-587. 1995.

BOHS, L.; OLMSTEAD, R.G. Phylogenetic relationships in Solanum (Solanaceae) based on ndhF sequences. Systematic Botany, Kent, v. 22, p. 5-17. 1997.

BOYES, S.; STRUBI, P. Organic acid and sugar composition of three New Zealand grown tamarillo varieties (Solanum betaceum (Cav.) New Zealand Journal of Crop and Horticultural Science, Wellington, v. 25, n.3, p.79-83, 1997.

CARDOSO, S. C.; SOARES, A. C. F.; BRITO, A. S. B.; CARVALHO, L. A.; PEIXOTO, C. C.; PEREIRA, M. E. C.; GOES, E. Qualidade de frutos de tomateiro com e sem enxertia. Fitotecnia, Campinas, v. 65, n. 2, p. 269-274, 2006.

CLARK, C.J.; RICHARDSON, A. C.. Biomass and mineral nutrient partitioning in a developing Tamarillo (Chyphomandra betacea) crop. Scientia Horticulturae, Amsterdam, v.94, n. 1, p.41-51, 2002.
DE ANGELIS, R. C. Fisiologia da nutrição: fundamentos para nutrição e desnutrição. São Paulo: EDART, 1977. v. 1, p. 43-53.

DIGNAN, C.; BURLINGAME, B.; KUMAR, S.; ALBERBERG, $\mathrm{W}$. The Pacific islands composition tables. 2 ed. Rome: FAO, 2004. 153p.

FRANCO, B.D.G.M.; LANDGRAF, M. Microbiologia dos alimentos. São Paulo: Ateneu, 1996.

INSTITUTO ADOLFO LUTZ. Normas analíticas do Instituto Adolfo Lutz: métodos químicos e físicos para análise de alimentos. São Paulo: EPU, 1985. 533p.

MWITHIGA, G.; MUKOLWE, M.I.; SHITANDA, D; KARANJA, P.N. Evaluation of the effect of ripening on the sensory quality and properties of tamarillo (Cyphomandra betaceae) fruits. Journal of Food Engineering, Kidlington, v.79, n.1, p. 117-123, 2007.

PROHENS, J.; NUEZ, F. The Tamarillo (Cyphomandra betacea). Small Fruits Review. Valencia, v.1, n. 2, p.43-68, 2005.

SOUThGATE, D. A. T. Determination of food carbohydrates. London: Applied Science Publishers, 1991. $177 \mathrm{p}$. 